\title{
Review
}

\section{Progression of Cognitive Decline in Parkinson's Disease}

\author{
Mandy Roheger ${ }^{\mathrm{a}}$, Elke Kalbe ${ }^{\mathrm{a}}$ and Inga Liepelt-Scarfone ${ }^{\mathrm{b}, \mathrm{c}, *}$ \\ ${ }^{a}$ Medical Psychology INeuropsychology and Gender Studies and Center for Neuropsychological Diagnostics \\ and Intervention (CeNDI), University Hospital Cologne, Köln, Germany \\ ${ }^{\mathrm{b}}$ German Center for Neurodegenerative Diseases (DZNE), University of Tübingen, Tübingen, Germany \\ ${ }^{\mathrm{c}}$ Department of Neurodegenerative Diseases, Hertie Institute for Clinical Brain Research, University of \\ Tübingen, Tübingen, Germany
}

Accepted 3 April 2018

\begin{abstract}
.
Background: Cognitive dysfunction is one of the most prevalent non-motor symptoms in Parkinson's disease (PD), often experienced as more debilitating for patients and caregivers than motor problems. Therefore, a deeper understanding of the course of cognitive decline and the identification of valid progression markers for Parkinson's disease dementia (PDD) is essential.

Objective: This systematic review summarizes the current state of knowledge on cognitive decline over time by reporting effect sizes of cognitive changes in neuropsychological tests.

Methods: 1368 studies were identified by a PubMed database search and 25 studies by additionally scanning previous literature. After screening all records, including 69 full-text article reviews, 12 longitudinal studies on the progression of cognitive decline in PD met our criteria (e.g., sample size $\geq 50$ patients).

Results: Only a few studies monitored cognitive decline over a longer period ( $>4$ years). Most studies focused on the evaluation of change in global cognitive state by use of the Mini-Mental State Examination, whereas the use of neuropsychological tests was highly heterogenic among studies. Only one study evaluated patients' cognitive performance in all specified domains (executive function, attention \& working memory, memory, language, and visual-spatial function) allowing for diagnosis of cognitive impairment according to consensus guidelines. Medium to strong effect sizes could only be observed in studies with follow-up intervals of four years or longer.

Conclusions: The results emphasize the need for the assessment of larger PD cohorts over longer periods of follow-up with a comprehensive neuropsychological battery.
\end{abstract}

Keywords: Parkinson's disease, cognition, longitudinal, progression, dementia

\section{INTRODUCTION}

Cognitive dysfunction is one of the most prevalent non-motor symptoms in Parkinson's disease

\footnotetext{
${ }^{*}$ Correspondence to: Inga Liepelt-Scarfone, Hertie Institute for Clinical Brain Research, University of Tübingen, HoppeSeyler Str. 3, 72076 Tübingen, Germany. Tel.: +49 07071 2980424; Fax: +49 07071 294490; E-mail: inga.liepelt@ uni-tuebingen.de.
}

(PD). Worsening of cognitive function up to Parkinson's disease dementia (PDD) can often be more debilitating for patients and their caregivers than the characteristic PD-related motor problems [1, 2]. Approximately $20 \%-33 \%$ of patients experience mild cognitive impairment (PD-MCI) already at the time of PD diagnosis [3, 4], and up to $60 \%-80 \%$ develop PDD within 12 years of disease duration [5]. In the clinical daily routine, the valid and early 
diagnosis of PDD is often difficult, as PD patients might not always be fully aware of their deficits $[6,7]$ and activity of daily living problems caused by cognitive impairment might not always be obvious for raters due to the presence of motor disabilities. Therefore, a deeper understanding of the natural course of cognitive decline and the identification of valid progression markers for PDD is of utmost importance for the early diagnosis and treatment of PDD.

The profile of cognitive dysfunction in PD patients is heterogeneous [8], but typically affects memory, attention, and executive as well as visual-spatial abilities [9]. To date, only a limited number of longitudinal studies exist that monitor cognitive impairment over the disease course in large PD samples. In a previous meta-analysis conducted in 2007 [10], Muslimovic and co-workers analysed data of 901 PD patients from 24 longitudinal studies to evaluate which cognitive domain is most affected by cognitive decline and to define the influence of demographic variables and disease duration on effect sizes of change. Results revealed that memory performance and visual-spatial abilities significantly worsen over time, whereas changes in further cognitive domains could not be verified. Older age and lower education levels were found to accelerate cognitive worsening, with more pronounced decline with longer follow-up intervals. However, one limitation was that most of the studies $(n=18,72 \%)$ reported in that review had a relatively small sample size of below 50 non-demented PD patients. Much research has been done since 2007, and data from longitudinal studies with larger sample sizes have been published. Therefore, this review aims at gathering the current state of knowledge on progression markers of cognitive decline, concentrating on the identification of cognitive tests that show the most decline over time. To ensure sufficient power for analysis, data is included from longitudinal studies with non-demented PD patients in which 50 PD patients or more were examined. Moreover, limitations of currently available data as a basis for recommendations on the design and data analysis of future studies on this topic are discussed.

\section{METHODS}

\section{Literature search}

As a basis, a manual search of papers included in the meta-analyses of Muslimovic et al. (2007) [10] was conducted, as the authors had the same research question and included papers published until
February 2007. Continuing our search, we used the identical search string used by Muslimovic and coworkers (2007) and reviewed papers from February 2007 to March 2017. The search was conducted in PubMed with the keywords Parkinsons disease or Parkinson's disease in combination with cognition, or cognitive impairment, or memory, or executive function, or neuropsychological tests, and longitudinal studies, or prognosis, or progression. Titles and abstracts were screened according to the eligibility criteria (see below). Afterwards, the full-text articles of the studies meeting the inclusion criteria were further reviewed for inclusion in the systematic review.

\section{Inclusion criteria}

To be included in the review, studies had to meet the following criteria: The articles had to be published in English or German, and a diagnosis of idiopathic PD had to be made according to validated clinical criteria. In contrast with the criteria specified by Muslimovic and collaborators, who included all sizes of PD samples, we decided to include only samples with at least 50 PD patients examined prospectively on at least two occasions with a minimum of one standardized neuropsychological test as a dependent variable (longitudinal design). In cases of several time points of measurement, only the first and the last are reported in this review. Neuropsychological test scores had to be presented for the PD group twice at baseline and at follow-up, or other statistics had to be reported that could be converted to effect sizes. In cases where different papers reported data concerning the same group of patients, the study with the largest sample or with the longest follow-up period was included.

\section{Exclusion criteria}

Reports published only in abstract form were excluded. Furthermore, clinical trials were not considered (e.g. deep brain stimulation or other pharmacological or non-pharmacological interventions studies) to examine the course of cognitive decline in PD without systematic effects.

\section{Outcome measures}

Tests for overall cognitive functioning and tests assessing specific cognitive domains were reported in the review. In Table 2, tests were assigned into one of six cognitive domains (overall cognition, memory, executive function, attention and working memory, 


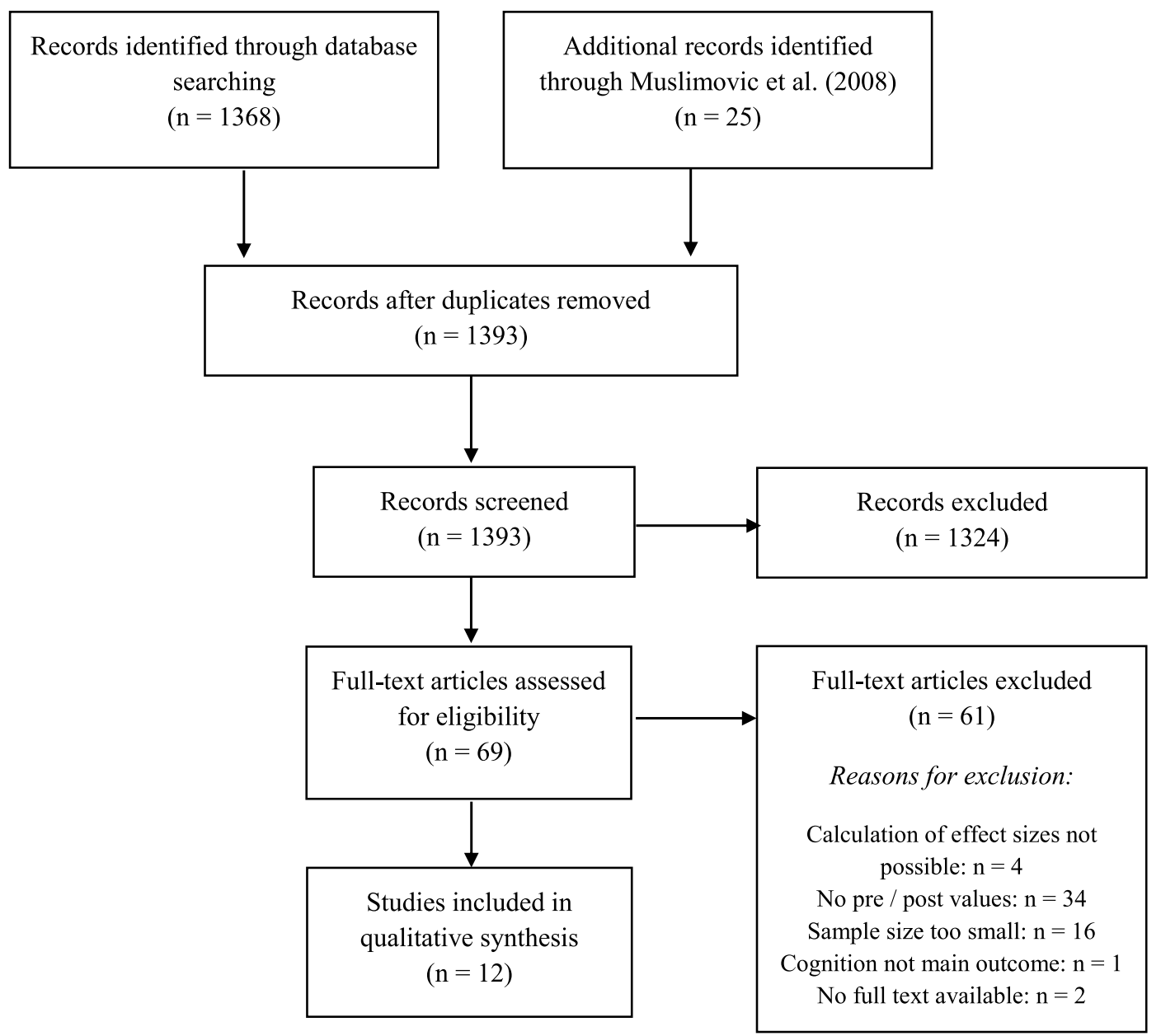

Fig. 1. Flow diagram of study selection process.

visual-spatial functions, and language) according to the definitions of Litvan and colleagues [11]. For tests that were not mentioned in the definitions of Litvan and co-workers, all authors conducted an expert rating to categorize the tests; in cases of heterogeneity, a consensus was made. In Table 3, assignment of tests to one specific cognitive domain referred to the descriptions of the authors in the reviewed studies if noted.

\section{Calculation of effect sizes}

From the data obtained in each study, the effect size Cohen's $d$ was calculated, indicating the mean difference between baseline and follow-up divided by the mean differences of the standard deviations at baseline and follow-up. A small effect is indicated when $|\mathrm{d}| \leq 0.2$, a medium effect is indicated when $|\mathrm{d}|$
$>0.2$ and $\leq 0.5$, and a large effect is indicated when $|\mathrm{d}|>0.5[12]$.

\section{RESULTS}

\section{Inclusion and exclusion process of the studies}

In total, 1368 studies were found through the database search and 25 studies by scanning the included studies in the systematic review and metaanalysis of Muslimovic and colleagues [10]. Figure 1 shows a PRISMA diagram illustrating the study selection process. After screening all records, 69 fulltext articles were assessed for eligibility. Of these, 34 $(49.3 \%)$ were excluded because they did not report pre- and post-values, $16(23.2 \%)$ were excluded because of a low sample size, in four $(5.8 \%)$ studies no calculation of effect sizes was possible, two 
(2.9\%) studies were excluded because no full-text was available, and in one study (1.4\%), cognition was not the main outcome and therefore insufficient data was available for calculation. Finally, $12(17.4 \%)$ studies were included in the review. All studies were published in English.

\section{Demographic and clinical characteristics of included studies}

An overview of study characteristics is outlined in Table 1. The baseline sample size varied greatly between studies from 60 [13] up to 1714 [14] PD patients. The mean age of the study sample was above 60 years in 10 out of 12 studies and ranged between 56.1 [15] and 70.0 years [16], with one study not reporting age at all [14].

Mean disease duration at baseline was heterogeneous with a range between 4 months [17] to 17.5 years [18]. Information on PD severity was given in all but three studies $[14,15,19]$. One study reported data for PD-MCI patients at baseline [17].

\section{Follow-up assessment}

The mean maximum follow-up times of the studies ranged from one year [17] to seven years [15]. Sample sizes of the included studies at follow-up measurement ranged from 49 patients [19] to 316 patients [14]. Three studies reported effect sizes separately for PD and PDD patients at time of follow-up assessment [15-17].

\section{Effect sizes of assessed cognitive domains}

Table 2 displays the effect sizes reported in the different studies according to the conducted neuropsychological tests in the assigned domain. Surprisingly, only one study evaluated patients' cognitive performance in all specified domains [20]. All other studies only evaluated specific aspects of cognitive worsening over time. Nine studies used screening tools to target overall cognition $[13,14,16-18,21$, 22]. Memory function was the most frequently targeted domain $(6 / 12 ;[13,15,18,20,22,23]$, followed by attention and working memory $(5 / 12 ;[13,15$, $18,20,22]$ and visual-spatial functions and language (both $5 / 12$ in the same studies; [15, 18, 20, 22, 23]. The domain executive function was only measured in 4 out of 12 studies [13, 18, 20, 23].

\section{Use of neuropsychological tests}

The choice of neuropsychological assessment was highly variable between the studies (see Table 3 for details). The most used screening tool for overall cognition was the MMSE in seven studies [13, 16-18, 21, 22]. The Wisconsin Card Sorting Test was assessed in four studies [13, 18, 20, 23]. Three studies assessed the Judgement of Line Orientation (JOLO) $[18,20,22]$ and the Wechsler Memory Scale [13, 20, $23]$. All other tests were only used in one or two studies. Furthermore, for nine neuropsychological tests, no information of assignment to a specific cognitive domain was given in the manuscripts.

\section{Effect sizes of neuropsychological tests}

Effect sizes of the neuropsychological tests show a large heterogeneity over studies according to duration of follow-up interval. Furthermore, as not every study assessed every domain, it is difficult to outline specific progression trends. However, as an important result, medium to strong effect sizes could only be observed in studies with follow-up intervals of four years or longer $[15,16,18]$. Effect sizes of the MMSE as a measure of global cognitive state were reported in seven studies with ranges for the effect sizes between -0.03 [17] and -1.39 [16], in patients with PDD. Lower score values in the MMSE, which are indicative for greater cognitive decline, were more likely with an increase of the follow-up period, meaning that the longer the follow up period, the greater the decline in the MMSE scores. Only one study showed an exception to this trend [18]; however, this study reported a higher dropout rate than the others.

\section{DISCUSSION}

The aim of this review was to provide an update on the current knowledge concerning the worsening of cognition in PD over time, and analyse which tests best monitor cognitive decline in PD. Based on our criteria, only 12 longitudinal studies could be identified. Of those, approximately $40 \%$ had longer follow-up intervals; for example, two studies with four years, one study each with five, six, and seven years of follow-up intervals. Most of the studies presented in this review focused on the evaluation of change in global cognitive status, primarily assessed with the MMSE, followed by the assessment of memory, attention, and working memory function over time. However, the use of neuropsychological tests 


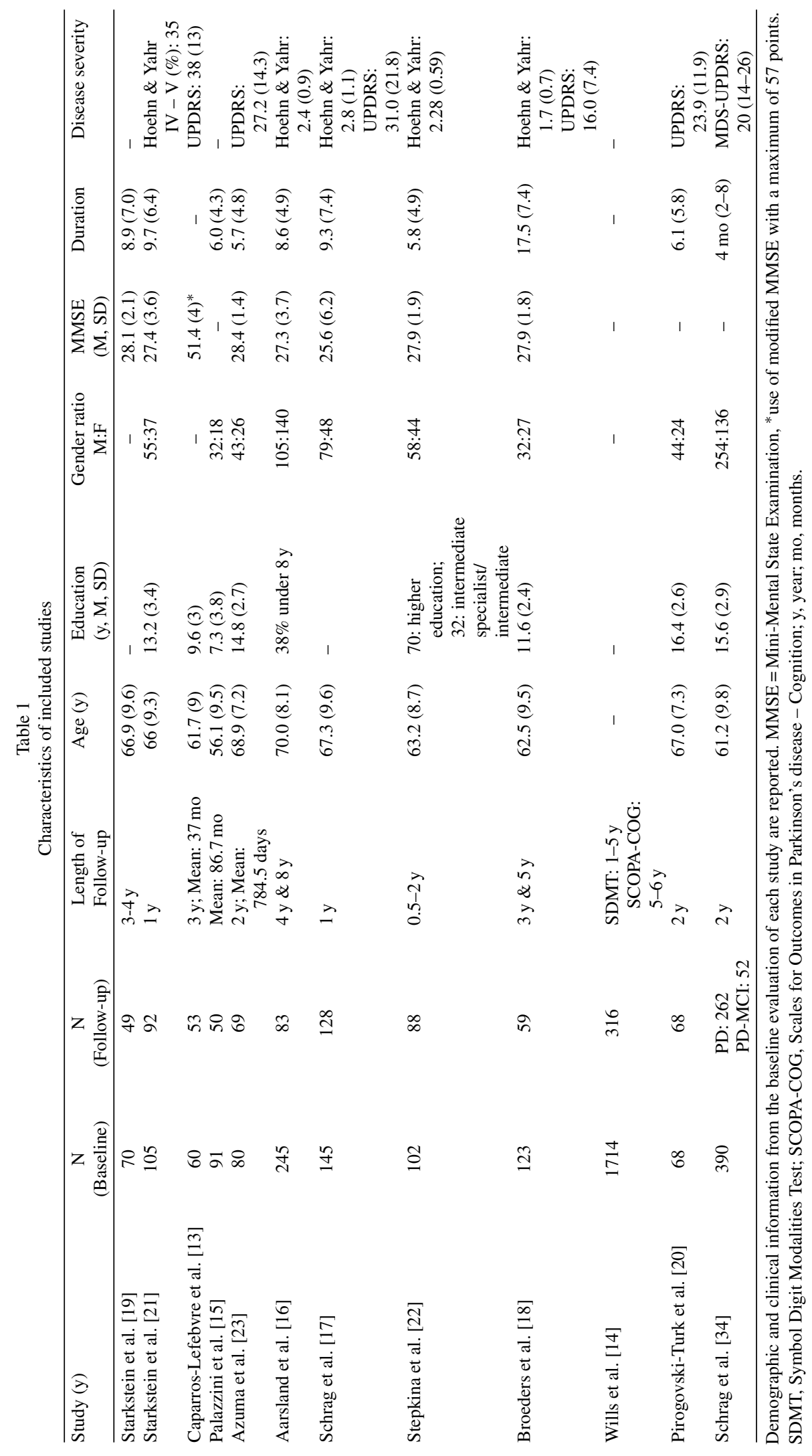




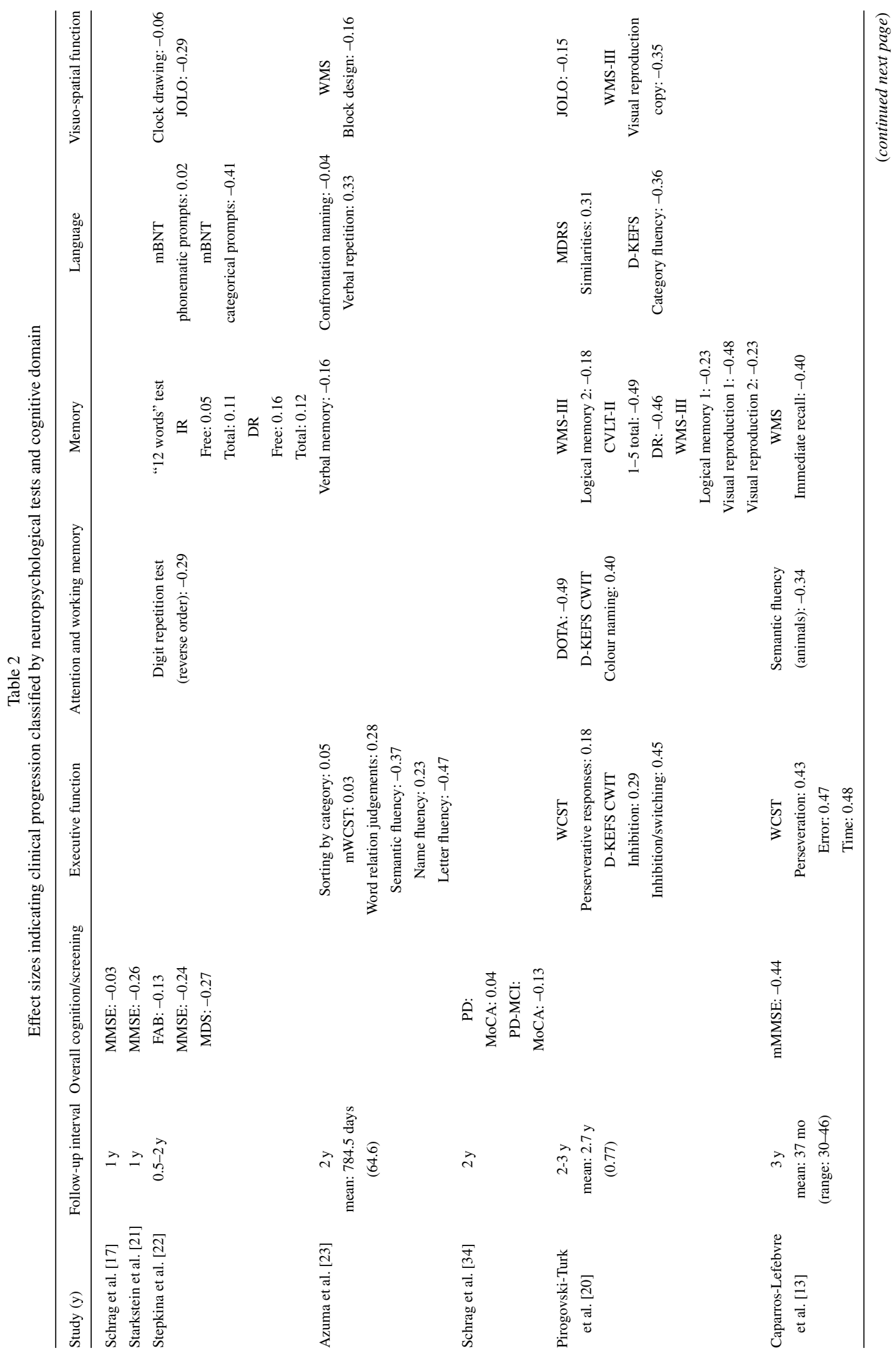




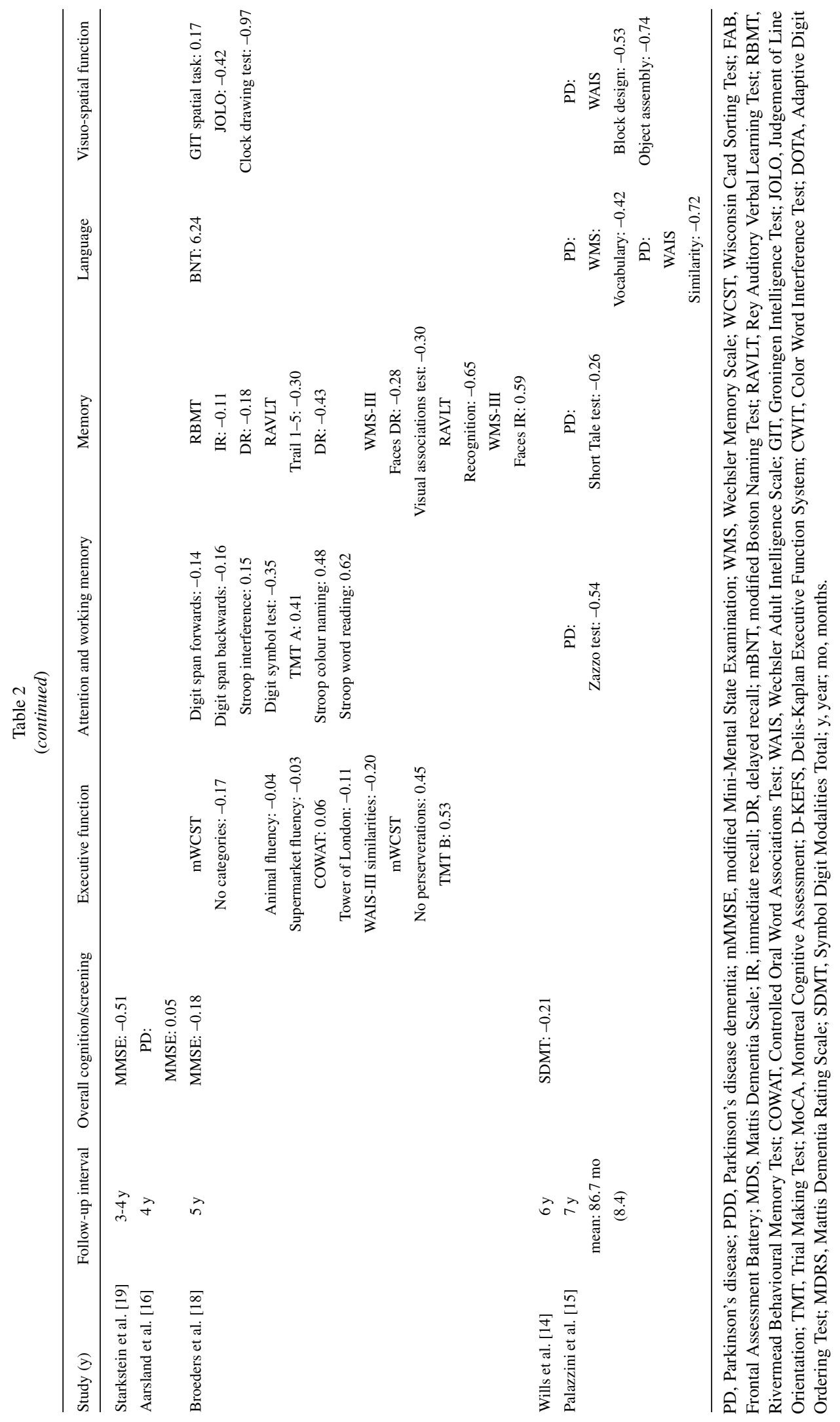


Table 3

Overview of used test, assigned domain in the reviewed studies and effect sizes

\begin{tabular}{|c|c|c|c|c|c|c|c|c|c|c|}
\hline $\begin{array}{l}\text { Domain assignment } \\
\text { according to } \\
\text { MDS-PD-MCI } \\
\text { criteria and expert } \\
\text { rating }\end{array}$ & Test & $\begin{array}{l}\bar{\pi} \\
\overline{0} \\
0 \\
0\end{array}$ & 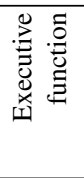 & 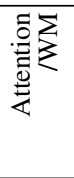 & $\begin{array}{l}\overrightarrow{0} \\
\stackrel{0}{0} \\
\sum_{2}^{0}\end{array}$ & 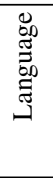 & 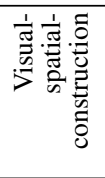 & 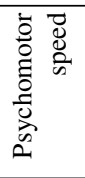 & 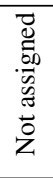 & $\begin{array}{c}\text { Effect sizes } \\
\text { (range) }\end{array}$ \\
\hline \multicolumn{11}{|l|}{ Overall cognition } \\
\hline & FAB & 1 & & & & & & & & -0.13 \\
\hline & MDS & 1 & & & & & & & & -0.27 \\
\hline & MMSE & 5 & & & & & & & 1 & $(-0.03)-(-1.39)$ \\
\hline & mMMSE & 1 & & & & & & & & -0.44 \\
\hline & $\mathrm{MoCa}$ & 1 & & & & & & & & $0.04-(-0.13)$ \\
\hline \multicolumn{11}{|l|}{ Executive function } \\
\hline & Animal fluency & & 1 & & & & & & & -0.04 \\
\hline & COWAT & & 1 & & & & & & & 0.06 \\
\hline & D-KEFS CWIT & & 1 & & & & & & & $0.29-0.45$ \\
\hline & Inhibition & & & & & & & & & \\
\hline & Inhibition/Switching & & & & & & & & & \\
\hline & mWCST & & & & & & & & 2 & 0.45 \\
\hline & Supermarket fluency & & 1 & & & & & & & -0.03 \\
\hline & Sorting by category & & & & & & & & 1 & 0.05 \\
\hline & Word relation judgements & & & & & & & & 1 & 0.28 \\
\hline & TMT B & & & 1 & & & & & & 0.53 \\
\hline & Tower of London & & 1 & & & & & & & -0.11 \\
\hline \multicolumn{11}{|c|}{ Attention \& working memory } \\
\hline & D-KEFS CWIT colour naming & & & 1 & & & & & & -0.36 \\
\hline & Digit span forwards & & & 1 & & & & & & -0.14 \\
\hline & Digit span backwards & & & 2 & & & & & & $(-0.16)-(-0.29)$ \\
\hline & DOTA & & & 1 & & & & & & -0.49 \\
\hline & Stroop interference & & & 1 & & & & & & 0.15 \\
\hline & TMT A & & & 1 & & & & & & 0.41 \\
\hline & Zazzo Test & & 1 & & & & & & & $(-0.54)-(-3.34)$ \\
\hline & Stroop colour naming & & & & & & & 1 & & 0.48 \\
\hline & Stroop word reading & & & & & & & 1 & & 0.62 \\
\hline & SDMT & & & 1 & & & & & & -0.21 \\
\hline \multicolumn{11}{|l|}{ Memory } \\
\hline & RAVLT DR & & & & 1 & & & & & -0.43 \\
\hline & RBMT DR & & & & 1 & & & & & -0.18 \\
\hline & Short Tale Test & & & & 1 & & & & & $(-0.26)-(-1.25)$ \\
\hline & Verbal memory & & & & 1 & & & & & -0.16 \\
\hline & WCST Perserverations & & 2 & & & & & & & $0.18-0.43$ \\
\hline & WMS-Immediate Recall & & & & 1 & & & & & -0.40 \\
\hline & WMS-III Logical memory 1 & & & & 1 & & & & & $(-0.23)-(-0.48)$ \\
\hline & Visual reproduction 1 & & & & & & & & & \\
\hline & Visual reproduction 2 & & & & & & & & & \\
\hline & " 12 words test", IR & & & & 1 & & & & & 0.11 \\
\hline & "12 words test", DR & & & & 1 & & & & & 0.12 \\
\hline \multicolumn{11}{|l|}{ Language } \\
\hline & BNT & & & & & 1 & & & & 6.24 \\
\hline & Confronting naming & & & & & & & & 1 & -0.04 \\
\hline & Letter fluency & & & & & & & & 1 & -0.47 \\
\hline & $\mathrm{mBNT}$ & & & & & & & & 1 & $0.02-(-0.41)$ \\
\hline & MDRS & & & & & 1 & & & & 0.31 \\
\hline & Name fluency & & & & & & & & 1 & 0.23 \\
\hline & Semantic fluency & & & & & & & & 1 & $(-0.34)-(-0.37)$ \\
\hline & Verbal repetition & & & & & & & & 1 & 0.33 \\
\hline & WAIS similarity & & & & & 1 & & & & -0.72 \\
\hline
\end{tabular}


Table 3

(Continued)

\begin{tabular}{|c|c|c|c|c|c|c|c|c|c|c|}
\hline $\begin{array}{l}\text { Domain assignment } \\
\text { according to } \\
\text { MDS-PD-MCI } \\
\text { criteria and expert } \\
\text { rating }\end{array}$ & Test & $\begin{array}{l}\overline{\bar{\pi}} \\
\overline{0} \\
0\end{array}$ & 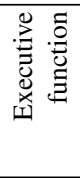 & 总是 & $\begin{array}{l}\overrightarrow{0} \\
\text { है } \\
\sum^{0}\end{array}$ & 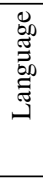 & 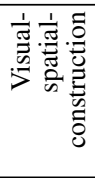 & 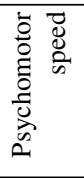 & 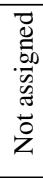 & $\begin{array}{c}\text { Effect sizes } \\
\quad \text { (range) }\end{array}$ \\
\hline \multicolumn{11}{|l|}{ Visual-spatial } \\
\hline & Clock drawing & & & & & & 2 & & & $(-0.06)-(-0.97)$ \\
\hline & GIT spatial task & & & & & & 1 & & & 0.17 \\
\hline & JOLO & & & & & & 3 & & & $(-0.15)-(-0.42)$ \\
\hline & Digit symbol test & & & & & & & 1 & & -0.35 \\
\hline & WAIS-III & & 1 & & & & & 1 & & -0.20 \\
\hline & WMS block design & & & & & & 1 & & & -0.16 \\
\hline
\end{tabular}

mMMSE, modified Mini-Mental State Examination; WMS, Wechsler Memory Scale; WCST, Wisconsin Card Sorting Test; FAB, Frontal Assessment Battery; MDS, Mattis Dementia Scale; IR, immediate recall; DR, delayed recall; mBNT, modified Boston Naming Test; RAVLT, Rey Auditory Verbal Learning Test; RBMT, Rivermead Behavioural Memory Test; COWAT, Controlled Oral Word Associations Test, WAIS, Wechsler Adult Intelligence Scale; GIT, Groningen Intelligence Test; JOLO, Judgement of Line Orientation; TMT, Trial Making Test; MoCA, Montreal Cognitive Assessment; D-KEFS, Delis-Kaplan Executive Function System; CWIT, Color Word Interference Test; DOTA, Adaptive Digit Ordering Test; MDRS, Mattis Dementia Rating Scale; SDMT, Symbol Digit Modalities Total.

was highly variable between the different studies, confirming previous reports comparing test assessments of European longitudinal PD studies [24]. Most importantly, only one study [20] evaluated patients' cognitive performance in all required domains for a level II diagnosis of PD-MCI and PDD based on the MDS Task Force criteria [25, 26]. Therefore, the data presented here emphasises the need for the assessment of larger PD cohorts over longer periods of follow-ups with a comprehensive neuropsychological battery.

Besides these limitations, our data confirms previous findings of the meta-analysis of Muslimovic and colleagues concerning studies with smaller samples in that more severe cognitive decline can be observed in studies with longer follow-up periods [10]. According to studies included in this review, medium to strong effect sizes could only be observed in studies with follow-up intervals of four years or longer $[15,16,18]$. Importantly, changes in cognitive performance were not limited to one specific cognitive domain, but included changes in various functions with longer follow-up intervals. Therefore, our data confirms previous reports arguing a slow and heterogenic progression of cognitive function in PD. The study of Broeders and co-workers revealed the highest effect in the language and visual-spatial domain after five years [18]. Worsening of visualspatial abilities has been reported to predict cognitive impairment and PDD [27, 28]. Previous studies have also shown that language problems occur in the transition to dementia in PD, with patients presenting problems in understanding and producing language, as well as impaired sentence comprehension [29]. However, higher order language problems and their cause are not yet well characterized in PD, which is currently best described as sentence comprehension and verbal fluency performance [30]. Spontaneous speech production and naming difficulties have been sparsely examined to date [31], but have been linked to cognitive impairment [32]. As underlying neuropathology of language problems executive-frontal related and temporal lobe dysfunction are discussed $[32,33]$, also affected in PD for example by loss of dopaminergic or cholinergic function, Lewy body or amyloid and taupathology.

Based on the high importance of understanding the course of cognitive decline up to dementia in PD, the number of the identified studies in this review seems to be surprisingly low. Most of the papers screened for eligibility in this review were primarily excluded by methodological reasons, as they did not present cognitive follow-up data.

To increase our understanding of the natural course of cognition in PD, large cohort studies with an elaborate neuropsychological test battery covering all relevant cognitive domains are needed. Moreover, reports should include both baseline and follow-up data to enable researchers to judge the progression of cognitive function over time. Only such studies would allow the identification of progression markers and different phenotypes for cognitive decline and the identification of tests able to predict cognitive decline and PDD with high accuracy. Finally, biomarker sampling in addition to comprehensive clinical assessment are important to identify the 
underlying pathomechanism of cognitive impairment in PD [34], further supporting different PD phenotypes and their risk of PDD within the disease course.

In summary, our results emphasize that cognitive functions in PD seem to underlie a slow progression which is heterogeneous and still not properly investigated. Therefore, our results show the need for larger PD cohorts that are examined over longer periods of follow-up with a comprehensive neuropsychological battery.

\section{CONFLICT OF INTEREST}

The authors have no conflict of interest to report.

\section{REFERENCES}

[1] Lawson RA, Yarnall AJ, Duncan GW, Breen DP, Khoo TK, Williams-Gray CH, Barker RA, Collerton D, Taylor J-P, Burn DJ (2016) Cognitive decline and quality of life in incident Parkinson's disease: The role of attention. Parkinsonism Relat Disord 27, 47-53.

[2] Leroi I, McDonald K, Pantula H, Harbishettar V (2012) Cognitive impairment in Parkinson's disease: Impact on quality of life, disability, and caregiver burden. $J$ Geriatr Psychiatry Neurol 25, 208-214.

[3] Lawson RA, Yarnall AJ, Duncan GW, Breen DP, Khoo TK, Williams-Gray CH, Barker RA, Burn DJ (2017) Stability of mild cognitive impairment in newly diagnosed Parkinson's disease. J Neurol Neurosurg Psychiatry 88, 648-652.

[4] Santangelo G, Vitale C, Picillo M, Moccia M, Cuoco S, Longo K, Pezzella D, di Grazia A, Erro R, Pellecchia MT (2015) Mild cognitive impairment in newly diagnosed Parkinson's disease: A longitudinal prospective study. Parkinsonism Relat Disord 21, 1219-1226.

[5] Hely MA, Reid WG, Adena MA, Halliday GM, Morris JG (2008) The Sydney multicenter study of Parkinson's disease: The inevitability of dementia at 20 years. Mov Disord 23, 837-844.

[6] Shulman LM, Pretzer-Aboff I, Anderson KE, Stevenson R, Vaughan CG, Gruber-Baldini AL, Reich SG, Weiner WJ (2006) Subjective report versus objective measurement of activities of daily living in Parkinson's disease. Mov Disord 21, 794-799.

[7] Leritz E, Loftis C, Crucian G, Friedman W, Bowers D (2004) Self-awareness of deficits in Parkinson disease. Clin Neuropsychol 18, 352-361.

[8] Kehagia AA, Barker RA, Robbins TW (2010) Neuropsychological and clinical heterogeneity of cognitive impairment and dementia in patients with Parkinson's disease. Lancet Neurol 9, 1200-1213.

[9] Aarsland D, Bronnick K, Williams-Gray C, Weintraub D, Marder K, Kulisevsky J, Burn D, Barone P, Pagonabarraga J, Allcock L, Santangelo G, Foltynie T, Janvin C, Larsen JP, Barker RA, Emre M (2010) Mild cognitive impairment in Parkinson's disease: A multicenter pooled analysis. Neurology 75, 1062-1069.

[10] Muslimović D, Schmand B, Speelman JD, De Haan RJ (2007) Course of cognitive decline in Parkinson's disease: A meta-analysis. J Int Neuropsychol Soc 13, 920-932.
[11] Litvan I, Goldman JG, Tröster AI, Schmand BA, Weintraub D, Petersen RC, Mollenhauer B, Adler CH, Marder K, Williams-Gray CH (2012) Diagnostic criteria for mild cognitive impairment in Parkinson's disease: Movement Disorder Society Task Force guidelines. Mov Disord 27, 349-356.

[12] Field A (2013) Discovering statistics using IBM SPSS statistics, Sage.

[13] Caparros-Lefebvre D, Pecheux N, Petit V, Duhamel A, Petit $\mathrm{H}$ (1995) Which factors predict cognitive decline in Parkinson's disease? J Neurol Neurosurg Psychiatry 58, 51-55.

[14] Wills A-MA, Elm JJ, Ye R, Chou KL, Parashos SA, Hauser RA, Bodis-Wollner I, Hinson VK, Christine CW, Schneider JS (2016) Cognitive function in 1736 participants in NINDS Exploratory Trials in PD Long-term Study-1. Parkinsonism Relat Disord 33, 127-133.

[15] Palazzini E, Soliveri P, Filippini G, Fetoni V, Zappacosta B, Scigliano G, Monza D, Caraceni T, Girotti F (1995) Progression of motor and cognitive impairment in Parkinson's disease. J Neurol 242, 535-540.

[16] Aarsland D, Andersen K, Larsen JP, Perry R, WentzelLarsen T, Lolk A, Kragh-Sørensen P (2004) The rate of cognitive decline in Parkinson disease. Arch Neurol 61, 1906-1911.

[17] Schrag A, Dodel R, Spottke A, Bornschein B, Siebert U, Quinn NP (2007) Rate of clinical progression in Parkinson's disease. A prospective study. Mov Disord 22, 938-945.

[18] Broeders M, Velseboer DC, de Bie R, Speelman JD, Muslimovic D, Post B, de Haan R, Schmand B (2013) Cognitive change in newly-diagnosed patients with Parkinson's disease: A 5-year follow-up study. J Int Neuropsychol Soc 19, 695-708.

[19] Starkstein SE, Bolduc PL, Mayberg HS, Preziosi TJ, Robinson RG (1990) Cognitive impairments and depression in Parkinson's disease: A follow up study. J Neurol Neurosurg Psychiatry 53, 597-602.

[20] Pirogovsky-Turk E, Moore RC, Filoteo JV, Litvan I, Song DD, Lessig SL, Schiehser DM (2017) Neuropsychiatric predictors of cognitive decline in Parkinson's disease: A longitudinal study. Am J Geriatr Psychiatry 25, 279-289.

[21] Starkstein SE, Mayberg HS, Leiguarda R, Preziosi TJ, Robinson RG (1992) A prospective longitudinal study of depression, cognitive decline, and physical impairments in patients with Parkinson's disease. J Neurol Neurosurg Psychiatry 55, 377-382.

[22] Stepkina D, Zakharov V, Yakhno N (2010) Cognitive impairments in progression of Parkinson's disease. Neurosci Behav Physiol 40, 61-67.

[23] Azuma T, Cruz RF, Bayles KA, Tomoeda CK, Montgomery EB (2003) A longitudinal study of neuropsychological change in individuals with Parkinson's disease. Int J Geriatr Psychiatry 18, 1115-1120.

[24] Lerche S, Liepelt-Scarfone I, Alves G, Barone P, Behnke S, Ben-Shlomo Y, Berendse H, Burn D, Dodel R, Grosset D, Heinzel S, Hu M, Kasten M, Kruger R, Maetzler W, Moccia M, Mollenhauer B, Oertel W, Roeben B, Sunkel U, Walter U, Wirdefeldt K, Berg D (2015) Methods in neuroepidemiology characterization of European longitudinal cohort studies in Parkinson's disease-report of the JPND Working Group BioLoC-PD. Neuroepidemiology 45, 282297.

[25] Litvan I, Goldman JG, Troster AI, Schmand BA, Weintraub D, Petersen RC, Mollenhauer B, Adler CH, Marder K, Williams-Gray CH, Aarsland D, Kulisevsky J, RodriguezOroz MC, Burn DJ, Barker RA, Emre M (2012) Diagnostic 
criteria for mild cognitive impairment in Parkinson's disease: Movement Disorder Society Task Force guidelines. Mov Disord 27, 349-356.

[26] Dubois B, Burn D, Goetz C, Aarsland D, Brown RG, Broe GA, Dickson D, Duyckaerts C, Cummings J, Gauthier S, Korczyn A, Lees A, Levy R, Litvan I, Mizuno Y, McKeith IG, Olanow CW, Poewe W, Sampaio C, Tolosa E, Emre M (2007) Diagnostic procedures for Parkinson's disease dementia: Recommendations from the movement disorder society task force. Mov Disord. 22, 2314-2324.

[27] Mahieux F, Fenelon G, Flahault A, Manifacier MJ, Michelet D, Boller F (1998) Neuropsychological prediction of dementia in Parkinson's disease. J Neurol Neurosurg Psychiatry 64, 178-183.

[28] Williams-Gray CH, Evans JR, Goris A, Foltynie T, Ban M, Robbins TW, Brayne C, Kolachana BS, Weinberger DR, Sawcer SJ, Barker RA (2009) The distinct cognitive syndromes of Parkinson's disease: 5 year follow-up of the CamPaIGN cohort. Brain 132, 2958-2969.

[29] Hobson P, Meara J (2004) Risk and incidence of dementia in a cohort of older subjects with Parkinson's disease in the United Kingdom. Mov Disord 19, 1043-1049.
[30] Robinson GA (2013) Primary progressive dynamic aphasia and Parkinsonism: Generation, selection and sequencing deficits. Neuropsychologia 51, 2534-2547.

[31] Ash S, Jester C, York C, Kofman OL, Langey R, Halpin A, Firn K, Perez SD, Chahine L, Spindler M (2017) Longitudinal decline in speech production in Parkinson's disease spectrum disorders. Brain Lang 171, 42-51.

[32] Berg E, Björnram C, Hartelius L, Laakso K, Johnels B (2003) High-level language difficulties in Parkinson's disease. Clin Linguist Phon 17, 63-80.

[33] Guidi M, Paciaroni L, Paolini S, Scarpino O, Burn DJ (2015) Semantic profiles in mild cognitive impairment associated with Alzheimer's and Parkinson's diseases. Funct Neurol 30, 113.

[34] Schrag A, Siddiqui UF, Anastasiou Z, Weintraub D, Schott JM (2017) Clinical variables and biomarkers in prediction of cognitive impairment in patients with newly diagnosed Parkinson's disease: A cohort study. Lancet Neurol 16, 66-75. 